Check for updates

Cite this: RSC Adv., 2018, 8, 17334

Received 29th January 2018

Accepted 14th March 2018

DOI: $10.1039 / \mathrm{c} 8 \mathrm{ra00888d}$

rsc.li/rsc-advances

\section{The unequivocal preponderance of biocomputation in clinical virology}

\author{
Sechul Chun, ${ }^{a}$ Manikandan Muthu, ${ }^{a}$ Judy Gopal, (D) a Diby Paul, ${ }^{b}$ Doo Hwan Kim, ${ }^{a}$ \\ Enkhtaivan Gansukh ${ }^{a}$ and Vimala Anthonydhason (D) *c
}

Bioinformatics and computer based data simulation and modeling are captivating biological research, delivering great results already and promising to deliver more. As biological research is a complex, intricate, diverse field, any available support is gladly taken. With recent outbreaks and epidemics, pathogens are a constant threat to the global economy and security. Virus related plagues are somehow the most difficult to handle. Biocomputation has provided appreciable help in resolving clinical virology related issues. This review, for the first time, surveys the current status of the role of computation in virus related research. Advances made in the fields of clinical virology, antiviral drug design, viral immunology and viral oncology, through input from biocomputation, have been discussed. The amount of progress made and the software platforms available are consolidated in this review. The limitations of computation based methods are presented. Finally, the challenges facing the future of biocomputation in clinical virology are speculated upon.

\section{Introduction}

Generally, viral outbreaks are pandemic and mostly transmitted via oral and nasal passages, the gastrointestinal tract, the skin and the urogenital tract/vagina. Viral diseases are not only a health threat, but they also result in outbreaks that have huge impacts on the global economy. Viral infections are a tremendous disease burden on humanity and combating them is an increasing challenge, since new viruses are continuously found. According to a recent World Health Organization (WHO) report, viral diseases which have recently caused outbreaks include: Ebola virus, influenza, human immunodeficiency virus (HIV), Middle East respiratory syndrome coronavirus (MERS-CoV), severe acute respiratory syndrome (SARS) and Zika viral infection. ${ }^{1}$ These diseases are major threats to health and global security. From 2013 to 2016, Ebola virus was most predominant in Guinea, Sierra Leone and Liberia. Since May 2016, 28616 suspected cases have been found, 11310 deaths reported and the fatality rate was approximately $70.8 \%{ }^{2,3}$ Outbreaks of Zika virus have been traced back to October 2015 in Brazil. The outbreak of Zika virus was evidenced by a rapid increase in cases among pregnant women, whose infants are born with extremely underdeveloped brains and will grow up to be adults

${ }^{a}$ Department of Environmental Health Science, Konkuk University, Seoul 143-701, Korea

${ }^{b}$ Environmental Microbiology, Department of Environmental Engineering, Konkuk University, Seoul 143-701, Korea

'Department of Biotechnology, Indian Institute of Technology-Madras, Chennai 600036, India. E-mail: vimalalisha@gmail.com; Fax: +91-44-22574102; Tel: +91-4422574101 with limited cognitive abilities and motor skills. The World Bank estimated that this outbreak caused a loss to the global economy to the magnitude of a total of $\$ 8.9$ billion USD. ${ }^{4}$ The identification and characterization of the causative agents and prophylaxis to limit the spread of a virus require the successful isolation of viral isolates via 'wet-lab' experimentation. This is necessary for curbing these outbreaks, in order to facilitate restoration.

\subsection{Importance of clinical virology}

With morbidity and mortality rates being significantly high with respect to virus related infections, clinical virology is at the forefront of research highlights. Clinical virology is a field of medicine which consists of the identification of viral pathogens responsible for human diseases like polio, chikungunya, severe acute respiratory disease (SARS), influenza, acquired immune deficiency syndrome (AIDS), Ebola hemorrhagic fever, hepatitis etc. ${ }^{5}$ Clinical virology is an interdisciplinary field, integrating virology/medical virology and healthcare sciences that characterize the safety and efficacy of medication, devices, diagnostic products and treatment regimes intended for mankind, which can be utilized for the prevention, treatment and diagnosis of infectious viral diseases. The effective prevention and clinical management of infectious diseases are intimately linked to the early and accurate screening of pathogens. This includes detecting the infectious particles in the organism and elucidating the aspects that confer resistance to therapy, mutations and genotype disparity. In this aspect, the accurate interpretation of laboratory results warrants the effective clinical management and control of a disease, ${ }^{6}$ however on the other 
hand, erroneous diagnosis could lead to financial and human loss. EM and culture-based methods work together and were one of the early traditional methods for the diagnosis of viral infections, along with serology testing for the detection of antibodies targeted against viruses. These conventional methods are still fundamental practices in many medical labs. ${ }^{7}$ Cell culturing is yet another popular method for isolating viruses using cell lines. ${ }^{8}$ The complement fixation test (CFT) is one of the oldest methods in the history of clinical virology. ${ }^{9}$ The haemagglutination inhibition test is generally used for detecting arboviruses and influenza and parainfluenza virus subtypes and is capable of yielding relative quantitation of the virus particles. ${ }^{\mathbf{1 0 , 1 1}}$ More recent, new generation diagnostic methods include: immunoassay methods, ${ }^{12,13}$ amplification based assays, ${ }^{14,15}$ mass spectrometric methods ${ }^{\mathbf{1 6 , 1 7}}$ and next generation sequencing..$^{\mathbf{1 8 , 1 9}}$

Biocomputational tools and database resources provide a wealth of valuable information about viral genomic sequences, molecular structures and viral-host pathogenesis. This information on infectious agents can lead to better diagnostics, therapeutics and vaccine development. Bioinformatics addresses these specific needs during data acquisition, storage and analysis and for the integration of this research with major therapeutic research areas such as viral oncology, viral immunology and antiviral research. In this review, the importance of exploring the role of biocomputation for additional knowledge in the realms of clinical virology is discussed. Advances made in the fields of clinical virology, antiviral drug design, viral immunology and viral oncology, through input from biocomputation, are recorded. Computation based approaches, their effects on clinical virology and their therapeutic usefulness are presented. Finally, the challenges facing the future of biocomputation in clinical virology are speculated upon.

\section{Current status of biocomputational approaches in clinical virology}

In virology, computational approaches have played crucial roles in various aspects of viral genome sequence analysis, therapeutic protein identification, anti-viral drug design/discovery, in silico vaccine design, differentially expressed gene identification, microRNA based signature identification and therapeutic design. Through in silico analysis, researchers gain a profound understanding of viral-host pathogenesis, which leads to better diagnostics, therapeutics and vaccines. Viruses have always been a major cause of a large number of infectious diseases. Molecular knowledge on viral proteins is thus seen to play an important role in the development of improved peptide-based vaccines, the design of novel anti-viral agents and the understanding of the entry mechanisms of viruses. Ongoing major research areas where biocomputation has played a positive role in clinical virology are represented in Fig. 1.

\subsection{Biocomputation for antiviral drug design}

Antiviral drugs are aimed at targeting selective viral infections. In particular, antiviral drugs have been designed against herpes, HIV, human cytomegalovirus (HCMV), varicella-zoster virus (VZV), hepatitis $\mathrm{B}$ and $\mathrm{C}$, and influenza $\mathrm{A}$ and $\mathrm{B}$ viral infections which have caused chronic infection in millions all over the world. ${ }^{20}$ There are many difficulties facing the accurate design of an antiviral drug against pathogenic viruses. Since viruses are parasites, they are unable to replicate on their own. They reproduce within the cells of an infected host and disturb the functions of the host cells. Many clinically important viruses don't have model systems due to their dangerous ability to mutate. Cultures are also hard to maintain and expensive. Viruses which show early infectious symptoms like influenza, common cold etc., are treated with antiviral drugs, but for viruses with symptoms that appear during the later stages of infection, the population is put at risk. ${ }^{21}$

A very early stage of viral infection is viral entry; a second approach is to target the processes that synthesize viral components after a virus invades a cell and establishes a critical infection. Based on the level of infection, the receptor protein and its therapeutic compounds are different. ${ }^{22}$ So before designing a drug, we need to ascertain the level of infection. To date, however, many viruses remain devoid of effective immunization and only a few antiviral drugs are licensed for clinical practice. Hence, there is an urgent need to discover novel antivirals that are highly efficacious and cost-effective for the management and control of viral infections at times when vaccines and standard therapies are lacking. ${ }^{23}$

Previously identified antiviral drugs do not destroy the target pathogen of the host, but instead they are able to reduce their growth and development. Modern in silico antiviral drug design aims to identify viral proteins (receptor proteins) that induce disease in humans or the survival of a microbial pathogen. Target proteins are common among many strains of viruses. Once the potent targets are identified, the appropriate drugs can be designed and administered. ${ }^{24}$ Target protein sequences were retrieved from sequence databases like viral genome databases (see Table 1) and structures will be retrieved from viral protein databases (Table 1). The binding site of the receptor protein is called the active site and this will be identified using in silico binding site prediction tools, such as active site prediction servers, CASTp, ${ }^{36}$ PDBeMotif, ${ }^{37}$ metaPocket, ${ }^{38}$ 3DLigandSite, ${ }^{39}$ Pocketome, ${ }^{40}$ PocketDepth, ${ }^{41}$ Pocket-Finder, ${ }^{42}$ FINDSITE $^{43}$ etc. Drugs can be designed which bind to the active region and inhibit receptor molecules. The structure of a drug molecule that can specifically interact with the biomolecules can be modeled using molecular modeling techniques via computational tools like I-TASSER, ${ }^{44}$ Robetta, ${ }^{45}$ HHpred, ${ }^{\mathbf{4 6}}$ MODELLER, ${ }^{47}$ MODBASE, ${ }^{48}$ RaptorX, ${ }^{49}$ SWISS-MODEL ${ }^{50}$ etc.

The first antiviral drug 5-iodo-2'-deoxyuridine (idoxuridine, IDU) was designed for the herpes virus infection in $1960 .^{51}$ Computer aided drug design (CADD) played an important role in designing a suitable antiviral drug against the deadly viral infection. There are two types of CADD, structure based and ligand based..$^{52}$ The available drugs were retrieved from antiviral drug databases (see Table 1). The successful design of antiviral drugs like Saquinavir, Relenza, and Tamiflu has validated the application of these techniques and indicates a bright future for drug discovery protocols. ${ }^{53}$ The creation of a structure based 


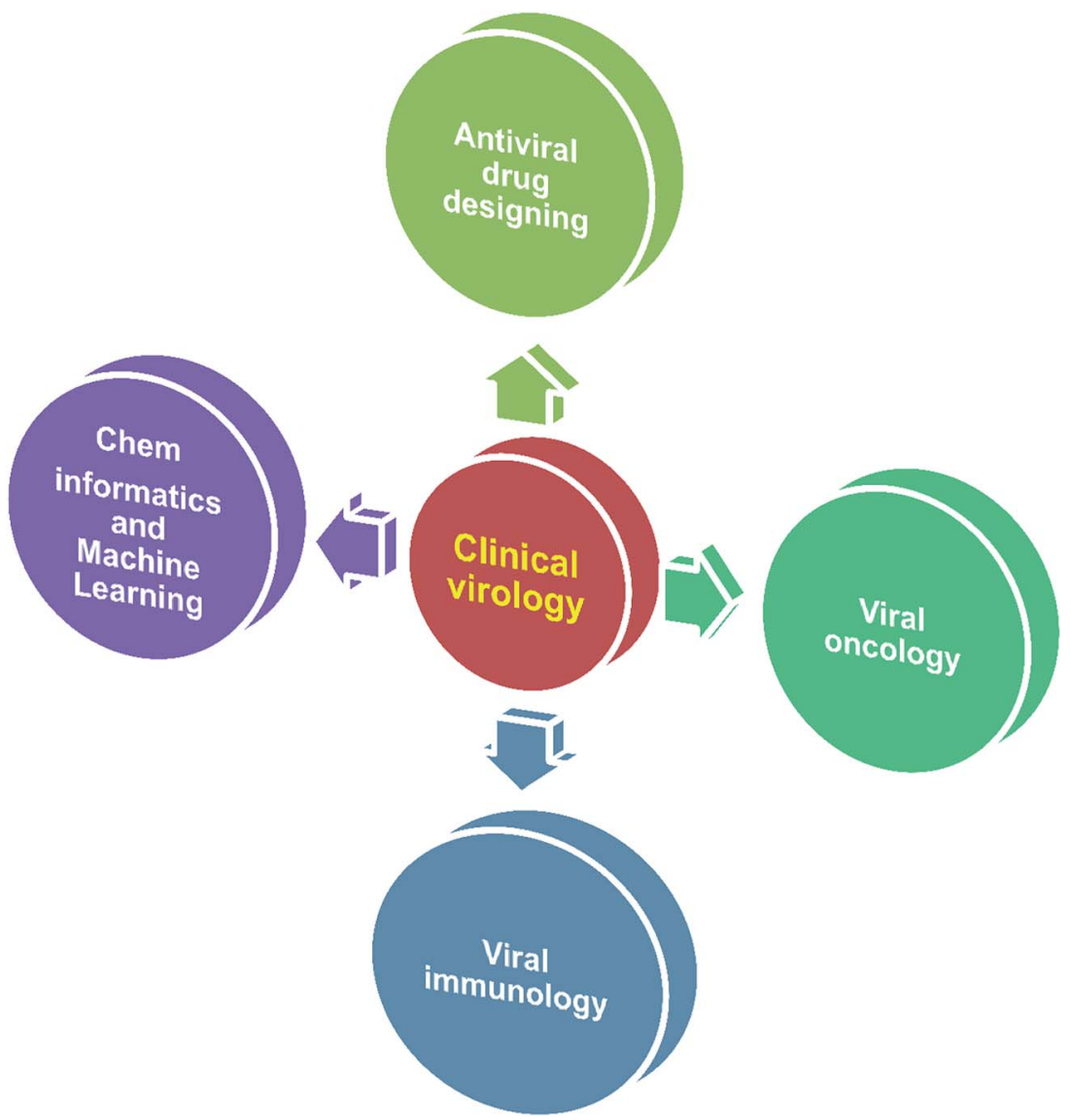

Fig. 1 Scheme depicting the ongoing major research areas in clinical virology.

pharmacophore model from the 3D structure of a target protein provides helpful information for investigating protein-ligand interactions and upgrading the knowledge of ligand binding affinities. The structure based pharmacophore regions are used for screening chemical databases for potential lead structures. CATALYST, ${ }^{54}$ PharmDock, ${ }^{55}$ PHASE, ${ }^{56}$ and ROCS $^{57}$ are used for pharmacophore modeling. Molecular docking is used to study the interactions between a target protein and a ligand molecule. Tools and software used for molecular docking include: AutoDock,,$^{58}$ DOCK, ${ }^{59}$ Glide, $^{60}$ GOLD, ${ }^{61}$ PatchDock ${ }^{62}$ etc. Molecular dynamics (MD) simulations can be used to provide dynamic insight into the structures of viruses and their components. ${ }^{63}$ These simulations can be performed using CHARMM ${ }^{64}$ Desmond, ${ }^{65}$ GROMACS ${ }^{66}$ etc. A flowchart for a typical antiviral drug design process is shown in Fig. 2.

\subsection{Biocomputation in viral immunology and subsequent in silico vaccine design}

Viral immunology is the most challenging and rapidly growing field in biology. During computational approaches, it relates to computational immunology or immunoinformatics, which deal with immunological problems using computational methods. ${ }^{67}$ Generally, the immune system connects thousands of molecules which become closely and intricately linked with each other; based on individuals the structure and the function of these molecules are different. Experimentally, immunologists have generated a vast amount of functional, clinical and epidemiological data. Using in silico techniques, these data have been stored and analyzed. In silico methods are affordable, because they reduce the time and costs involved in the laboratory analysis of pathogenic proteins. In vivo methods require the cultivation of the pathogenic genome in order to identify antigenic proteins. Although pathogens grow quickly, the extraction of their proteins and then the testing of those proteins on a large scale is expensive and time consuming. ${ }^{68}$ The use of immunoinformatics solves these problems and can identify virulence genes, potential target proteins and binding sites; this will lead to the development of novel vaccines and immunotherapeutics. This in silico method for vaccine design is called reverse vaccinology.

In 1796, Edward Jenner first identified the smallpox vaccine and introduced the term vaccine..$^{69}$ A century later, Louis Pasteur introduced a rational method for vaccine development. ${ }^{70}$ Subsequently, researchers discovered many in vivo vaccines like the polio vaccine (Albert Sabin), ${ }^{71}$ measles, mumps and rubella (Hilleman), ${ }^{72}$ and so on. In later years, researchers faced the challenge of cultivating pathogenic viruses. It was found that in the case of hepatitis B viral infection, the pathogenic virus could not be cultured in vitro. Thus, they collected the viral antigen from chronically infected patients, the antigens were inactivated and the vaccine was developed. ${ }^{73}$ At the end of the $20^{\text {th }}$ century, researchers developed vaccines based on the genomes 
Table 1 A consolidated list of various biocomputational resources used in clinical virology

\begin{tabular}{|c|c|c|c|}
\hline Feature & Computational resource & Website & Reference \\
\hline \multirow[t]{6}{*}{ Viral sequence database } & Viral genomes resource & https://www.ncbi.nlm.nih.gov/genome/viruses/ & 25 \\
\hline & $\begin{array}{l}\text { Virus pathogen database and } \\
\text { analysis resource (ViPR) }\end{array}$ & https://www.viprbrc.org/ & 26 \\
\hline & $\begin{array}{l}\text { Viral genome databases } \\
\text { (VGDB) - Oxford academic }\end{array}$ & https://www.oxfordjournals.org/nar/database/subcat/5/18 & 27 \\
\hline & $\begin{array}{l}\text { Viral reference sequences - } \\
\text { ViralZone }\end{array}$ & http://www.viralzone.expasy.org/6096 & 28 \\
\hline & viruSITE & http://www.virusite.org/ & 30 \\
\hline & RNAVirusDB & $\begin{array}{l}\text { http://virus.zoo.ox.ac.uk/rnavirusdb } \\
\text { http://hivweb.sanbi.ac.za/rnavirusdb } \\
\text { http://bioinf.cs.auckland.ac.nz/rnavirusdb } \\
\text { http://tree.bio.ed.ac.uk/rnavirusdb }\end{array}$ & 31 \\
\hline $\begin{array}{l}\text { Viral protein structure } \\
\text { database }\end{array}$ & VPDB & http://vpdb.bicpu.edu.in/ & 32 \\
\hline & $\begin{array}{l}\text { Drug office - oral } \\
\text { antiviral drugs }\end{array}$ & $\begin{array}{l}\text { https://www.drugoffice.gov.hk/eps/do/en/consumer/ } \\
\text { news_informations/dm_17.html }\end{array}$ & 103 \\
\hline & $\begin{array}{l}\text { Virus pathogen database and } \\
\text { analysis resource (ViPR) }\end{array}$ & https://www.viprbrc.org/ & 26 \\
\hline & $\begin{array}{l}\text { Database of anticancer peptides } \\
\text { and } \\
\text { proteins (CancerPPD) }\end{array}$ & http://crdd.osdd.net/raghava/cancerppd/ & 104 \\
\hline & $\begin{array}{l}\text { Cancer drug resistance } \\
\text { database (CancerDR) }\end{array}$ & http://crdd.osdd.net/raghava/cancerdr/ & 105 \\
\hline & CancerHSP & http://lsp.nwsuaf.edu.cn/CancerHSP.php & 106 \\
\hline & & http://www.inpacdb.org & 107 \\
\hline
\end{tabular}

of microorganisms. The first pathogenic organism identified by the reverse vaccinology approach was meningococcus B. ${ }^{74}$ Recently, Reza Taherkhani and Fatemeh Farshadpour developed an epitope based vaccine for the hepatitis $\mathrm{E}$ viral infection $^{75}$ and vaccines have been designed for other recent outbreaks including for the Zika virus ${ }^{76}$ and the Ebola virus. ${ }^{77}$
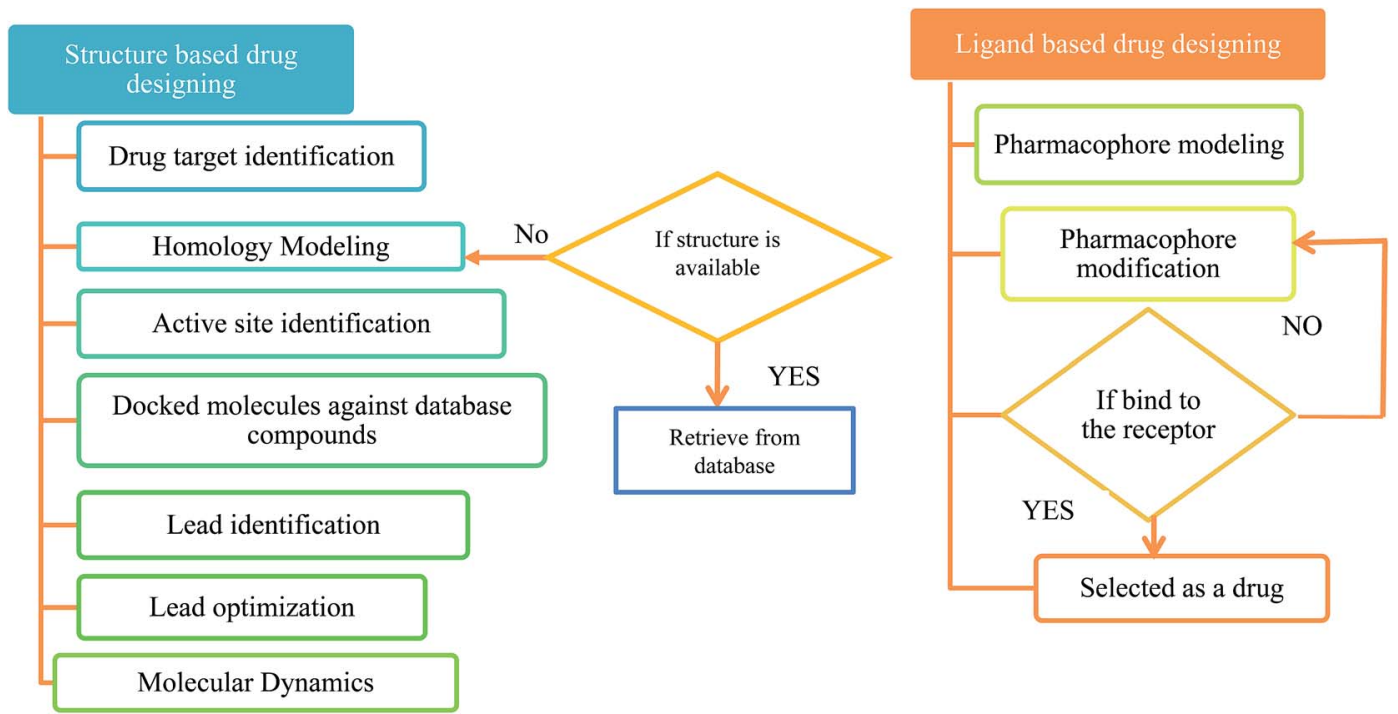

Fig. 2 A flowchart for antiviral drug design. 
An adaptive immune response occurs against a pathogen. There are two types of adaptive immune response, cellular and humoral, mediated by $\mathrm{T}$ cells and $\mathrm{B}$ cells. The antigenic determinant region is called the epitope, which is recognized by the corresponding receptor present on $\mathrm{B}$ or $\mathrm{T}$ cells. ${ }^{78}$ In bioinformatics, epitope based vaccine design relies on machine learning techniques, such as support vector machines (SVMs), hidden Markov models (HMMs) and neural networks (NNs). ${ }^{79-81}$ The in silico vaccine design process requires the identification of a key molecule on the receptor protein, which is predicted using the Epitope Database (IEDB).$^{82}$ Then, the immunogenetic peptides will be identified using Kolaskar and Tongaonkar's predicted antigenic peptide tools, based on applied semi empirical methods, ${ }^{83}$ and the peptide-epitope will be mapped. Subsequently, peptide and immunodominant epitope docking will be performed for vaccine design. Molecular dynamics based simulations will be carried out for conformational analysis. Finally, the peptide will be synthesized for practical application. The steps involved in in silico vaccine design are shown in Fig. 3.

\subsection{Biocomputational inputs in viral oncology}

Viral oncology is an ongoing and essential research field for biologists. According to a report in 2017 by the American Cancer Society, 1688780 cancer cases were expected to be treated in
$2017 .{ }^{84}$ Certain tumors are reported to be caused by viruses like the human papilloma virus (HPV), Epstein-Barr virus (EBV), Kaposi's sarcoma-associated herpesvirus (KSHV), hepatitis C virus (HCV), human immunodeficiency virus (HIV) and hepatitis $B$ virus (HBV). More than $90 \%$ of anal cancers have been caused by $500000 \mathrm{HPV}$ infections per year worldwide. ${ }^{85}$ Traditionally, viral oncology research has relied on biological techniques, but recently, computational techniques have enabled the diagnosis of malignancies.

In recent years, high throughput technologies have generated vast amounts of data, but on the other hand only limited experimental information is available for most genes. Next Generation Sequencing (NGS) plays an especially important role in cancer therapeutics. Computers can analyze vast amounts of data using remarkable techniques based on supercomputers. Computational oncology techniques have created in silico biological system models. To understand normal vs. malignant patient sequence characteristics, bioinformaticians first retrieved enormous sequences from various databases. ${ }^{86}$ Tumor suppressor genes (TSG), sequence annotation, their relation to diseases and gene ontology (GO) processes are identified using these databases. Cellular pathway databases have played an important role in identifying the essential proteins that induce disease, constructing biological networks and predicting models. ${ }^{87}$ Using traditional methods, only single genotype-phenotype relationships can be

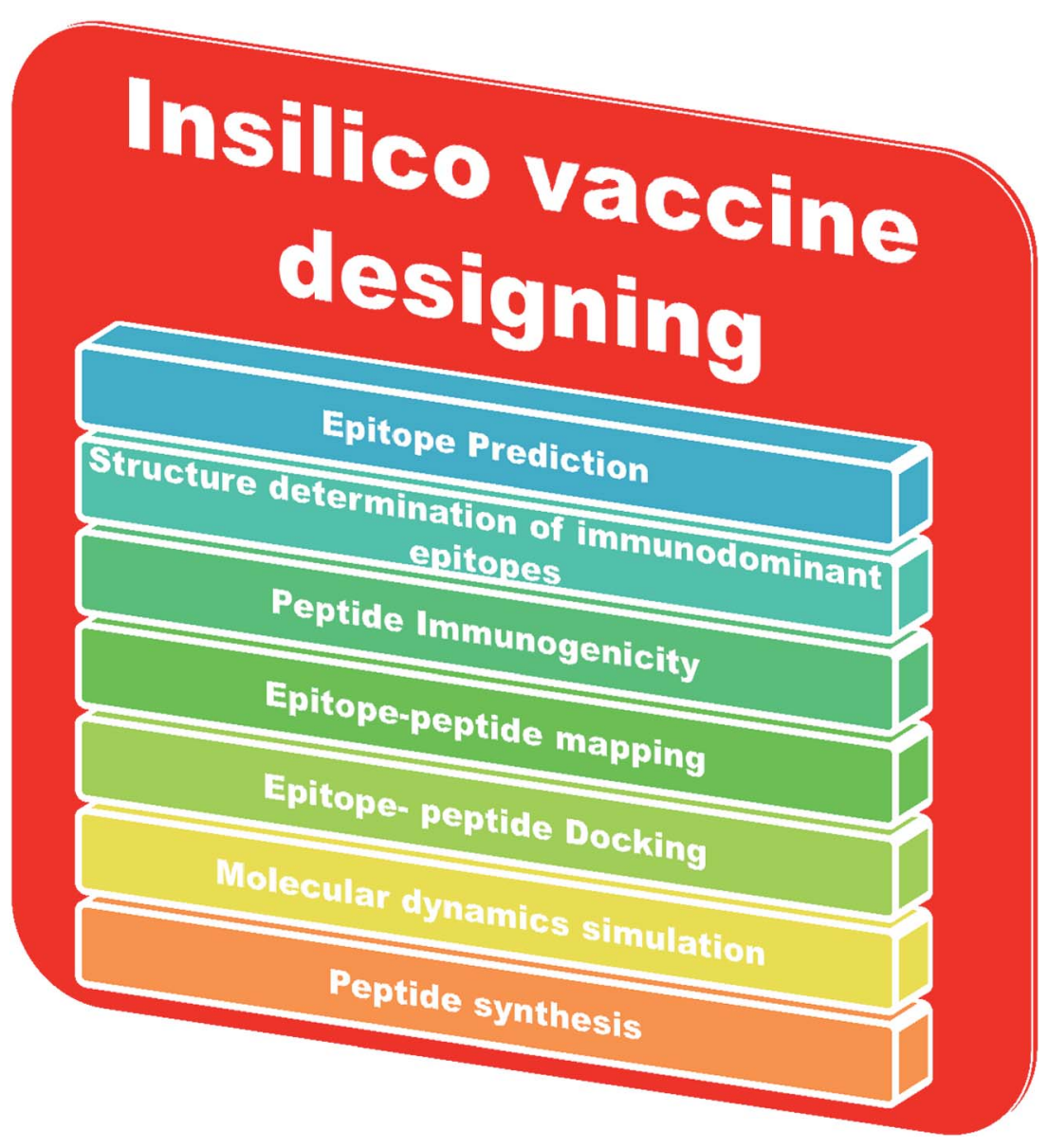

Fig. 3 Steps in in silico vaccine design. 
identified at a time. In contrast, high-throughput technology examines the phenotypic outcomes of multiple mutations simultaneously. ${ }^{88}$ Fig. 4 presents the typical process steps of the NGS of clinical data.

Microarrays contain thousands of gene expressions and transcriptome data. Using a computer, we can analyse microarray data. From transcriptome data, differentially expressed genes can be identified using NGS techniques. The differentially expressed genes lead to the identification of cellular target genes that identify the therapeutic compound. ${ }^{\mathbf{8 9} 90}$ Computer based drug design techniques use the pharmacokinetics and pharmacodynamic relationships of the available anticancer agents to improve treatment and drug development. These techniques include virtual screening, QSAR (quantitative structure activity relationship) models and molecular docking. ${ }^{91}$ Pharmacophore-mapping algorithms are employed for the inverse screening of some representative compounds for a large set of pharmacophore models constructed from human target proteins. Molecular docking studies were carried out to assess the binding affinity of these compounds to proteins responsible for mediating tumor growth. Furthermore, the important structural features of compounds for anticancer activity were assessed using Monte Carlo-based SMILES and hydrogen graph-based QSAR studies. ${ }^{92}$

\subsection{Cheminformatics and machine learning models for anti- viral drug discovery}

Structure activity relationships (SARs) with molecular systems, quantitative structure activity relationships (QSARs) and

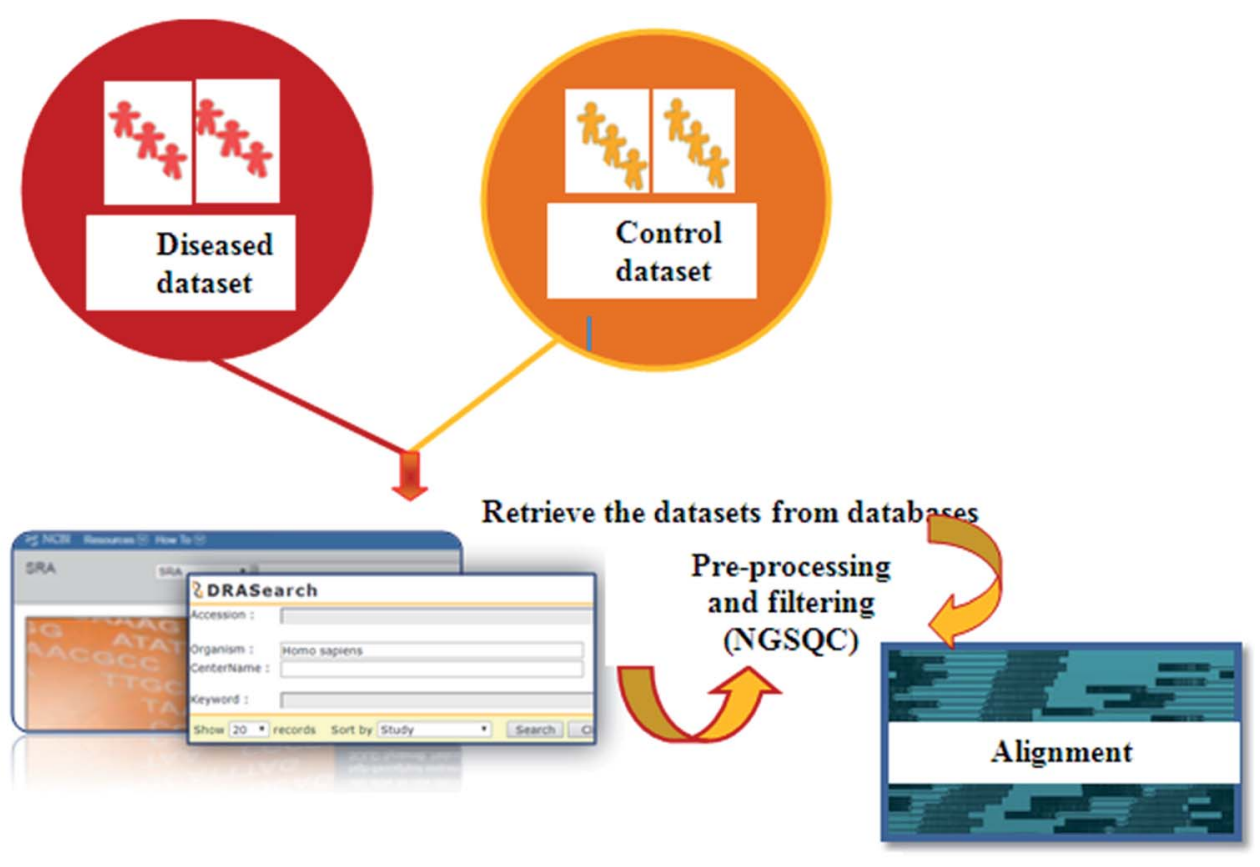

Divergent Analysis of NGS data

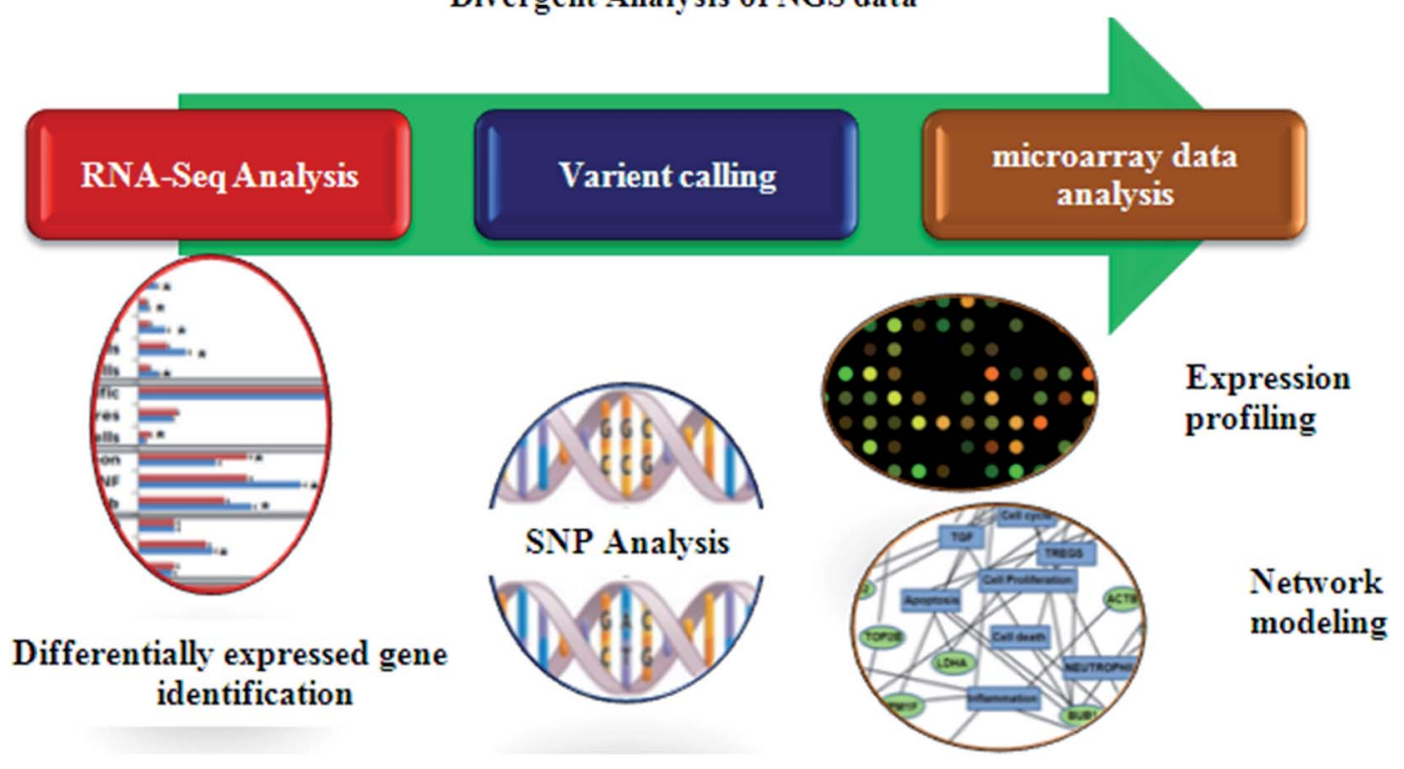

Fig. 4 Schematic representation of the process steps of the NGS of clinical data. 
quantitative structure-property relationships (QSPRs) with molecules play a major role in computational drug discovery. In this context, machine learning approaches for viral drug discovery are pioneering as an emerging and ongoing research technique in clinical virology. Machine learning approaches can help make complex network models for confirming the effectiveness of combinations of drugs for epidemiological outbreaks in large populations. In 2015, Herrera and his coworkers collected datasets from ChEMBL (anti-HIV chemical compounds), the AIDSVu database (HIV surveillance reports) and the Census Bureau (socioeconomic data) and proposed the first artificial neural network (ANN) model for the prediction of HAART cocktails, to halt AIDS in the epidemic networks of the U.S. ${ }^{93}$ The first multitasking model for quantitative structurebiological effect relationships (mtk-QSBERs) was predicted, in silico fragment based drug design for drug-molecule interaction study was performed and its molecular entities were screened by virtual screening for the Hepatitis $\mathrm{C}$ viral infection. This was then experimentally validated for anti-HCV activity and ADMET (Absorption, Distribution, Metabolism, Excretion and Toxicity) properties. ${ }^{94}$ Very recently, the first multitasking model was developed for anti-HIV agents from 29682 HIV cases using quantitative structure-biological effect relationships (mtk-QSBER) based on fragment based drug design and virtual screening approaches. More than $96 \%$ of fragments contributed towards the multiple biological effects. ${ }^{95}$

ANNs have been used to link data related to AIDS in the U.S. to ChEMBL data. ANNs are network prediction models that are mainly used in medicinal chemistry and drug development. ${ }^{\mathbf{9 6}}$ Moreover, in 2008 Francisco and coworkers constructed drugdrug complex networks against different species of virus. ${ }^{97}$ González-Díaz et al., used the MARkovian CHemicals IN SIlico DEsign (MARCH-INSIDE) approach and predicted novel antimicrobial drugs and targets using drug-drug similarity complex networks. ${ }^{98}$ Computational studies of structural stability relationships produce novel stochastic moments. In 2005, González-Díaz et al. constructed a new Markov model, which makes use of novel stochastic moments such as molecular descriptors for viral protein surfaces in quantitative structureactivity relationship (QSAR) studies for small molecules for human rhinoviruses (HRVs). ${ }^{99}$ Markovian Backbone Negentropies (MBNs) have been introduced in order to model their effects on protein structure stability relationships. An MBN based on a Markov chain model of electron delocalization throughout the protein backbone for the computational study of structure/stability relationships has also been reported. ${ }^{\mathbf{1 0 0}}$ These approaches mainly focus on cheminformatic approaches towards drug design, drug development and drug-target interactions in in silico clinical fields, especially with respect to viral diseases.

\subsection{Challenges facing biocomputation in clinical virology}

Antiviral drug discovery and design processes are not exceptionally without fault; they do have certain limitations and face a few challenges. Firstly, therapeutic target selection is difficult

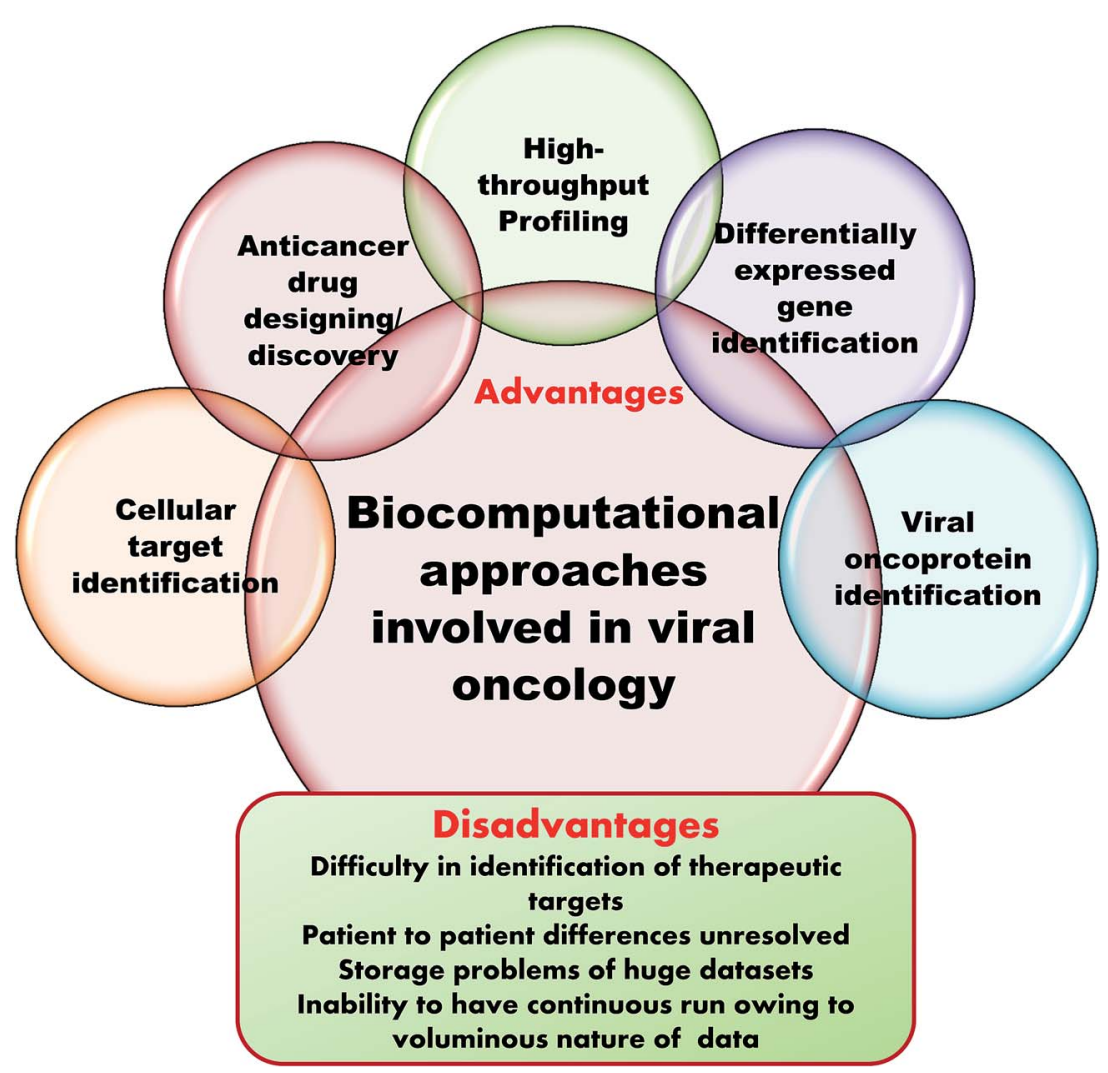

Fig. 5 Advantages and disadvantages of biocomputation in clinical virology. 
in certain conditions, for instance in the disordered physiological processes (pathophysiology) of nervous system related disorders. Thus, the integration of large experimental data with machine learning approaches requires the development of new brain-inspired computational algorithms. ${ }^{110}$ If the molecular mechanisms of the disease are unknown, it is difficult to find the specific receptor and with the function of the protein not determined, it is difficult to make much progress. ${ }^{111}$ No single medicine is a common solution for all diseased patients; it may vary based on the patient's symptoms, disease conditions and their history. In such a case, we have to follow a personalized medicinal approach. Based on this approach we need gene expression data for individuals in order to identify the potent drug target and design a drug and in such situations we need a knowledge base. We need to investigate many drug targets based on the drug candidate, which is a trial and error process. ${ }^{112}$ The use of molecular signatures like peptides in epitope based vaccine design hits a limitation here due to a lack of delivery systems or disease models, rendering it incapable of reaching its set goals in therapeutics. ${ }^{113}$ The mtk-QSBER model was able to integrate multiple chemical compounds with multiple biological target molecules and develop drug like compounds. ${ }^{93,94,114-116}$

Emerging diseases, especially viral diseases including cancer related viruses, threaten human life with mortality and morbidity. Emerging viral diseases caused by RNA viruses are increasing due to increasing mutation rates. In clinical virology, emerging techniques, such as microarrays (expression analysis), metagenomic biosynthetic gene cluster identification, host-pathogen interaction analysis, unknown diseaseassociated viruses, the discovery of novel human viruses and NGS technologies (differential gene expression, de novo sequencing, epigenetics, variant calling, SNPs etc.), are enormously supported. ${ }^{117,118}$ Using these technologies, it is possible to analyze millions of data simultaneously at a low cost.

Microarrays generate a vast amount of gene expression datasets for healthy and control samples. NGS also produces gigabytes of transcriptome data. Here, the major challenge is storage of this voluminous data. This requires multiprocessors and multicore computers with hundreds of gigabytes of RAM and terabytes of hard drive space in Linux operating systems. Even with the use of internet based supercomputing, the delivery of results is greatly delayed and results become queued up. It is also impossible to submit whole datasets as these have to be divided up to adhere to the accessible memory size and then submitted. In this way, the entire process is greatly affected and becomes time consuming because of the data storage issue. ${ }^{119}$ Fig. 5 summarizes the advantages and disadvantages of computation in clinical virology.

\section{Summary}

Computer-assisted clinical virology research areas are without doubt very essential, although many aspects of improvement are still in progress. Every year, lots of web-assisted tools, software and algorithms are continuously being developed for various aspects of antiviral drug design, viral immunology and viral oncology. High throughput technologies generate data for thousands of patients as well as their corresponding expression data. Thus, computer-assisted approaches do overcome cumbersome wet lab procedures to a large extent and provide valuable insights into clinical virology and a positive direction for antiviral drug design, epitope-based vaccine design, differentially expressed gene identification and potential drug target identification strategies.

\section{Conflicts of interest}

The authors have no conflicts of interest whatsoever.

\section{References}

1 Emergencies preparedness, response, WHO report 2017,http://www.who.int/csr/don/archive/year/2017/en/.

2 B. Jeremy, H. Jonas and M. Timothy, et al., The economics of Ebola, LSE Connect I, summer, 2015, pp. 11-13.

3 WHO Ebola Response Team, Ebola Virus Disease in West Africa - The First 9 Months of the Epidemic and Forward Projections, N. Engl. J. Med., 2014, 371(16), 1481-1495.

4 P. Aparaajita, Economic Cost of Zika Virus ICWA View Point, 2016.

5 W. James L. Duc and N. Nathanson, Emerging Viral Diseases. Why We Need to Worry about Bats, Camels, and Airplanes. Emergence and Control of Viral Infections, 2016.

6 S. M. Lemon, M. A. Hamburg and P. F. Sparling, et al., Global infectious disease surveillance and detection: assessing the challenges-finding solutions, Workshop Summary, Institute of Medicine (US) Forum on Microbial Threats, National Academies Press, US, 2007.

7 C. S. Goldsmith and S. E. Miller, Modern uses of electron microscopy for detection of viruses, Clin. Microbiol. Rev., 2009, 22(4), 552-563.

8 F. C. Robbins, J. F. Enders and T. H. Weller, Cytopathogenic effect of poliomyelitis viruses in vitro on human embryonic tissues, Exp. Biol. Med., 1950, 75(2), 370-374.

9 J. Casals and R. Palacios, The complement fixation test in the diagnosis of virus infections of the central nervous system, J. Exp. Med., 1941, 74(5), 409-426.

10 M. Grandien, C. A. Pettersson, L. Svensson, et al., Latex agglutination test for adenovirus diagnosis in diarrheal disease, J. Med. Virol., 1987, 23(4), 311-316.

11 R. Sandeep, K. Rajesh, R. A. Antony, et al., Rapid particle agglutination test for human immunodeficiency virus: hospital-based evaluation, J. Clin. Microbiol., 2002, 40(4), 1553-1554.

12 M. Deguchi, N. Yamashita, M. Kagita, et al., Quantitation of Hepatitis B surface antigen by an automated chemiluminescent microparticle immunoassay, J. Virol. Methods, 2004, 15, 217-222.

13 N. Shinkai, K. Matsuura, F. Sugauchi, et al., Application of a newly developed high-sensitivity HBsAg chemiluminescent enzyme immunoassay for Hepatitis B patients with HBsAg seroclearance, J. Clin. Microbiol., 2013, 51(11), 3484-3491. 
14 K. B. Mullis and F. A. Faloona, Specific synthesis of DNA in vitro via a polymerase-catalysed chain reaction, Methods Enzymol., 1987, 155, 335-350.

15 R. J. Hall, M. Peacey, Q. S. Huang, et al., Rapid method to support diagnosis of swine-origin influenza virus infection by sequencing of real-time PCR amplicons from diagnostic assays, J. Clin. Microbiol., 2009, 47, 3053-3054.

16 J. K. Lewis, M. Bendahmane, T. J. Smith, R. N. Beachy and G. Siuzdak, Identification of viral mutants by mass spectrometry, Proc. Natl. Acad. Sci. U. S. A., 1998, 95(15), 8596-8601.

17 G. Siuzdak, Probing viruses with mass spectrometry, J. Mass Spectrom., 1998, 33, 203-211.

18 F. Sanger, S. Nicklen and A. R. Coulson, DNA sequencing with chain-terminating inhibitors, Proceedings of the National Academy of Science USA, 1977, 74, 5463-5467.

19 S. N. Naccache, S. Federman, N. Veeraraghavan, M. Zaharia, D. Lee, E. Samayoa, J. Bouquet, A. L. Greninger, K. C. Luk, B. Enge, D. A. Wadford, S. L. Messenger, G. L. Genrich, K. Pellegrino, G. Grard, E. Leroy, B. S. Schneider, J. N. Fair, M. A. Martínez, P. Isa, J. A. Crump, J. L. DeRisi, T. Sittler, J. Hackett Jr, S. Miller and C. Chiu, A cloudcompatible bioinformatics pipeline for ultra-rapid pathogen identification from next-generation sequencing of clinical samples, Genome Res., 2014, 24(7), 1180-1192.

20 E. De Clercq, Antivirals and antiviral strategies, Nat. Rev. Microbiol., 2004, 2, 704-720.

21 A. L. Menéndez and F. Gago, Antiviral Agents: Structural Basis of Action and Rational Design, Subcell. Biochem., 2013, 68, 599-630.

22 E. M. Damm and L. Pelkmans, Systems biology of virus entry in mammalian cells, Cell. Microbiol., 2006, 8, 1219-1227.

23 L. T. Lin, W. C. Hsu, C. C. Lin, et al., Antiviral Natural Products and Herbal Medicines, Journal of Traditional and Complementary Medicine, 2014, 4(1), 24-35.

24 A. D. Welch, P. Calabresi, W. H. Prusoff, et al., Initial Clinical Studies with 5-Iodo-2'-deoxyuridine, Experimental Cell Research, 1963, 24, 479.

25 B. J. Rodney, A. Danso, B. Yiming, et al., NCBI Viral Genomes Resource, Nucleic Acids Res., 2014, 3, 1, DOI: 10.1093/nar/gku1207.

26 E. P. Brett, S. Douglas, Y. Z. Greer, et al., Virus Pathogen Database and Analysis Resource (ViPR): A Comprehensive Bioinformatics Database and Analysis Resource for the Coronavirus Research Community, Viruses, 2012, 4, 32093226.

27 H. C. U. David, Viral Genome DataBase: storing and analyzing genes and proteins from complete viral genomes, Bioinformatics, 2000, 16(5), 484-485.

28 C. Hulo, E. Castro, P. Masson, et al., ViralZone: a knowledge resource to understand virus diversity, Nucleic Acids Res., 2011, 39, D576-D582.

29 Z. Yun and B. D. Aevermann, Influenza Research Database: An integrated bioinformatics resource for influenza virus research, Nucleic Acids Res., 2017, 45, D466-D474.

30 S. Matej, B. Gabor, K. Lubos, et al., viruSITE-integrated database for viral genomics, Database, 2016, baw162.
31 B. Robert, O. Tulio, M. Sidney, et al., The RNA Virus Database, Nucleic Acids Research, 2009, 37(1), D431-D435.

32 O. P. Sharma, A. Jadhav, A. Hussain, et al., VPDB: Viral Protein Structural Database, Bioinformation, 2011, 6(8), 324-326.

33 M. M. Alba, D. Lee and F. M. Pearl, VIDA: a virus database system for the organization of animal virus genome open reading frames, Nucleic Acids Res., 2001, 29(1), 133-136.

34 C. M. Shepherd, I. A. Borelli, G. Lander, et al., VIPERdb: a relational database for structural virology, Nucleic Acids Res., 2006, 34, D386-D389.

35 L. Palmeira, S. Penel, V. Lotteau, et al., PhEVER: a database for the global exploration of virus-host evolutionary relationships, Nucleic Acids Res., 2011, 39, D569-D575.

36 L. Jie, E. Herbert and W. Clare, Anatomy of Protein Pockets and Cavities: Measurement of Binding Site Geometry and Implications for Ligand Design, Protein Sciene, 1998, 7, 1884-1897.

37 PD. BeMotif Tutorial, Protein Data Bank in Europe, http:// pdbe.org/motif/.

38 A. Golovin and K. Henrick, MSDmotif: exploring protein sites and motifs, BMC Bioinf., 2008, 9, 312.

39 B. Huang, MetaPocket: a meta approach to improve protein ligand binding site prediction., OMICS, 2009, 13(4), 325330.

40 M. N. Wass and L. A. Kelley, Sternberg MJ. 3DLigandSite: predicting ligand-binding sites using similar structures, Nucleic Acids Res., 2010, 38, W469-W473.

41 J. An, M. Totrov, R. Abagyan, et al., Pocketome via comprehensive identification and classification of ligand binding envelopes, Mol. Cell. Proteomics, 2005, 4, 752-761.

42 Y. Kalidas and N. Chandra, PocketDepth: a new depth based algorithm for identification of ligand binding sites in proteins, J. Struct. Biol., 2008, 161(1), 31-42.

43 K. Theodora, A. Georgios, G. Spyroulias, et al., Computational approaches in target identification and drug discovery, Comput. Struct. Biotechnol. J., 2016, 14, 177-184.

44 J. Yang, R. Yan and A. Roy, The I-TASSER Suite: Protein structure and function prediction, Nat. Methods, 2015, 12, 7-8.

45 E. K. David, C. Dylan and B. David, Protein structure prediction and analysis using the Robetta server, Nucleic Acids Res., 2004, 32, W526-W531.

46 S. Johannes, B. Andreas, N. Andrei, et al., The HHpred interactive server for protein homology detection and structure prediction, Nucleic Acids Res., 2015, 33(1), W244W248.

47 A. Sali and T. L. Blundell, Comparative protein modelling by satisfaction of spatial restraints, J. Mol. Biol., 1993, 234(3), 779-815.

48 U. Pieper, N. Eswar, A. C. Stuart, et al., MODBASE, a database of annotated comparative protein structure models, Nucleic Acids Res., 2002, 30(1), 255-259.

49 K. Morten, W. Haipeng, W. Sheng, et al., Template-based protein structure modeling using the RaptorX web server, Nat. Protoc., 2012, 7, 1511-1522. 
50 M. Biasini, S. Bienert, A. Waterhouse, et al., SWISS-MODEL: modelling protein tertiary and quaternary structure using evolutionary information, Nucleic Acids Res., 2014, 42(W1), W252-W258.

51 H. Reuveni, C. O. Bull, M. L. Landry, et al., Antiviral activity of 5-iodo-2'-deoxyuridine and related drugs in human keratinocytes infected in vitro with herpes simplex virus type 1, Skin Pharmacol., 1991, 4(4), 291-297.

52 K. Vikash, C. Sharat, I. Mohammad, et al., Recent Advances in the Development of Antiviral Agents Using Computeraided Structure Based Approaches, Curr. Pharm. Des., 2014, 20(21), 3488-3499.

53 L. Backert and O. Kohlbacher, Immunoinformatics and epitope prediction in the age of genomic medicine, Genome Med., 2015, 7(119), DOI: 10.1186/s13073-015-02450 .

54 A. H. Evan, D. Chaya, et al., Use of Catalyst Pharmacophore Models for Screening of Large Combinatorial Libraries, $J$. Chem. Inf. Comput. Sci., 2002, 0042(5), 1204-1211.

55 H. Bingjie and M. A. Lill, PharmDock: a pharmacophorebased docking program, J. Cheminf., 2014, 6, 14.

56 S. L. Dixon, A. M. Smondyrev and E. H. Knoll, PHASE: a new engine for pharmacophore perception, 3D QSAR model development, and 3D database screening: Methodology and preliminary results, J. Comput.-Aided Mol. Des., 2006, 20(10-11), 647-671.

57 T. S. Rush, J. A. Grant, L. Mosyak, et al., A shape-based 3-D scaffold hopping method and its application to a bacterial proteinprotein interaction, J. Med. Chem., 2005, 48(5), 1489-1495.

58 G. M. Morris, R. Huey and A. J. Olson, Using AutoDock for ligandreceptor docking, Curr. Protoc. Bioinformatics, 2008, 8(14).

59 M. Shingo and D. Irwin, Automated flexible ligand docking method and its application for database search, J. Comput. Chem., 1997, 18(14), 1812-1825.

60 R. A. Friesner, J. L. Banks, R. B. Murphy, et al., Glide: a new approach for rapid, accurate docking and scoring Method and assessment of docking accuracy, J. Med. Chem., 2004, 47(7), 1739-1749.

61 G. Jones, P. Willett and R. C. Glen, Molecular recognition of receptor sites using agenetic algorithm with a description of desolvation, J. Mol. Biol., 1995, 245(1), 43-53.

62 S. D. Dina, I. R. Yuval, J. Haim, et al., PatchDock and SymmDock: servers for rigid and symmetric docking, Nucleic Acids Res., 2005, 33(2), W363-W367.

63 R. Tyler and S. P. S. Mark, Computational virology: From the inside out, Biochim. Biophys. Acta, 2016, 7, 1610-1618.

64 B. R. Brooks, C. L. Brooks, A. D. Mackerell, et al., CHARMM: The biomolecular simulationprogram, J. Comput. Chem., 2009, 30(10), 1545-1614.

$65 \mathrm{~J}$. B. Kevin and C. Edmond, Scalable Algorithms for Molecular Dynamics Simulations on Commodity Clusters (PDF), Proceedings of the ACM/IEEE Conference on Supercomputing (SC06), Tampa, Florida, November 11-17, 2006.
66 N. Schmid, A. Eichenberger, A. Choutko, et al. Definition and testing of the GROMOS force-field, Eur. Biophys. J., 2011, 40(7), 843-856.

67 L. Backert and O. Kohlbacher, Immunoinformatics and epitope prediction in the age of genomic medicine, Genome Med., 2015, 7, 119, DOI: 10.1186/s13073-0150245-0.

68 N. Rapin, O. Lund, M. Bernaschi, et al. Computational Immunology Meets Bioinformatics: The Use of Prediction Tools for Molecular Binding in the Simulation of the Immune System, PLoS One, 2010, 5(4), e9862.

69 M. N. Davies and D. R. Flower, Harnessing bioinformatics to discover new vaccines, Drug Discovery Today, 2007, 12(9-10), 389-395.

70 L. Pasteur, De l'attenuation du virus du Choléra des poules, C. R. Seances Acad. Sci., 1880, 91, 673-680.

71 M. M. Levine, R. Lagos and J. Esparza, Vaccines and Vaccination in Historical Perspective, New Generation Vaccines, Informa Healthcare Inc., New York, USA, 4th edn, 2009, pp. 1-11.

72 P. A. Offit. Vaccinated: One Man's Quest to Defeat the World's Deadliest Diseases, HarperCollins, New York, 2007.

73 E. B. Buynak, R. R. Roehm, A. A. Tytell, et al., Vaccine Against Human Hepatitis B, JAMA, J. Am. Med. Assoc., 1996, 276(22), 1793-1795.

74 M. M. Giuliani, J. Adu-Bobie, M. Comanducci, et al., A universal vaccine for serogroup B meningococcus, Proc. Natl. Acad. Sci., 2006, 103(29), 10834-10839.

75 T. Reza and F. Fatemeh, A new strategy for development of hepatitis E vaccine: Epitope-based vaccines, Pathog. Infect. Dis., 2015, 1, e933, DOI: 10.14800/pid.933.

76 A. Alam, S. Ali and S. Ahamad, From ZikV genome to vaccine: in silico approach for the epitope-based peptide vaccine against Zika virus envelope glycoprotein, Immunology, 2016, 149(4), 386-399, DOI: 10.1111/ imm.12656.

77 R. Dash, R. Das and M. Junaid, In silico-based vaccine design against Ebola virus glycoprotein, Adv. Appl. Bioinf. Chem., 2017, 21(10), 11-28, DOI: 10.2147/AABC.S115859.

78 K. Thomas, J. Goldsby and J. Kuby et al., Kuby Immunology, WH Freeman and Co, New York, 6th edn, 2006.

79 M. Bhasin and G. P. Raghava, Analysis and prediction of affinity of TAP binding peptides using cascade SVM, Protein Sci., 2004, 13(3), 596-607.

80 J. E. P. Larsen, O. Lund and M. Nielsen, Improved method for predicting linear B-cell epitopes, Immunome Res., 2006, 2(2), DOI: 10.1186/1745-7580-2-2.

81 S. Saha and G. P. Raghava, Prediction of continuous B-cell epitopes in an antigen using recurrent neural network, Proteins, 2006, 65(1), 40-48.

$82 \mathrm{~J}$. Greenbaum, The Immune Epitope Database and Analysis Resource (IEDB), EMBRACE Bioinformatics of Immunology Workshop, La Jolla Institute for Allergy and Immunology, Lyngby, Denmark, January 24, 2007.

83 A. S. Kolaskar and P. C. Tongaonkar, A semi-empirical method for prediction of antigenic determinants on 
protein antigens, FEBS Lett., 1990 Dec 10, 276(1-2), 172174.

84 Cancer facts and figures 2017, American Cancer society, https://www.cancer.org/content/dam/cancer-org/research/ cancer-facts-and-statistics/annual-cancer-facts-and-figures/ 2017/cancer-facts-and-figures-2017.pdf.

85 I. H. Frazer, D. R. Lowy and J. T. Schiller, Prevention of cancer through immunization: Prospects and challenges for the 21st century, Eur. J. Immunol., 2007, 37(1), S148S155.

86 A. W. C. Daskalaki and R. P. T. Herwig, Computational tools and resources for systems biology approaches to cancer, Comput. Biol., 2009, 227-242.

87 D. Janusz, K. Michael and A. Michal, A gene ontology inferred from molecular networks, Nat. Biotechnol., 2013, 31(1), 37-48.

88 C. Nicholas and P. Y. Arthur, High-throughput profiling of influenza A virus hemagglutinin gene at single-nucleotide resolution, Sci. Rep., 2015, 4, 4942.

89 D. V. Nguyen and D. M. Rocke, On partial least squares dimension reduction for microarray based classification, Comput. Stat. Data Anal., 2004, 46, 407-425.

90 Y. Chen, D. Nguyen and T. Pham, Identification of relevant genes from microarray experiments based on partial least squares weights: application to cancer genomics, Computational Biology: Issues and Applications in Oncology, New York, Springer, 2009, pp. 1-18.

91 A. V. Denisenko, T. Druzhenko and Y. Skalenko, Photochemical synthesis of 3 azabicyclo[3.2.0] heptanes: advanced building blocks for drug discovery, J. Org. Chem., 2017, 82(18), 9627-9636.

92 B. Chalyk, M. Butko and O. Yanshyna, Synthesis of spirocyclic pyrrolidines: advanced building blocks for drug discovery, Chem.-Eur. J, 2017, 23(66), 16782-16786.

93 D. M. Herrera-Ibatá, A. Pazos, R. A. Orbegozo-Medina, et al. Mapping chemical structure-activity information of HAART-drug cocktails over complex networks of AIDS epidemiology and socioeconomic data of U.S. counties, Biosystems, 2015, 132-133, 20-34.

94 A. Speck-Planche, Dias Soeiro Cordeiro MN. Speeding up Early Drug Discovery in Antiviral Research: A FragmentBased in Silico Approach for the Design of Virtual AntiHepatitis C Leads, ACS Comb. Sci., 2017, 19(8), 501-512.

95 V. V. Kleandrova and A. Speck Planche, Multitasking model for computer-aided design and virtual screening of compounds with high anti-HIV activity and desirable ADMET properties, in. Multi-Scale Approaches in Drug Discovery: From Empirical Knowledge to In Silico Experiments and Back, ed. A. Speck-Planche, Elsevier, Oxford, UK, 2017, pp. 55-81.

96 H. González-Díaz, D. M. Herrera-Ibatá, A. Duardo-Sánchez, et al. ANN multiscale model of anti-HIV drugs activity $v s$. AIDS prevalence in the US at county level based on information indices of molecular graphs and social networks, J. Chem. Inf. Model., 2014, 54(3), 744-755.

97 F. J. Prado-Prado, O. Martinez de la Vega, E. Uriarte, et al. Unified QSAR approach to antimicrobials. 4. Multi-target
QSAR modeling and comparative multi-distance study of the giant components of antiviral drug-drug complex networks, Bioorg. Med. Chem., 2009, 17(2), 569-575.

98 H. González-Díaz, F. Prado-Prado and F. M. Ubeira, Predicting antimicrobial drugs and targets with the MARCH-INSIDE approach, Curr. Top. Med. Chem., 2008, 8(18), 1676-1690.

99 H. González-Díaz and E. Uriarte, Biopolymer stochastic moments. I. Modeling human rhinovirus cellular recognition with protein surface electrostatic moments, Biopolymers, 2005, 77(5), 296-303.

100 R. Ramos de Armas, H. González Díaz, R. Molina, et al. Markovian Backbone Negentropies: Molecular descriptors for protein research. I. Predicting protein stability in Arc repressor mutants, Proteins, 2004, 56(4), 715-723.

101 Department of health drug office drug registration and import/export control division guidance notes on registration of pharmaceutical products/substances, 2017, 1-12.

102 B. Dominique, C. Joseph and L. Bruno, Computational oncology - mathematical modelling of drug regimens for precision medicine, Nat. Rev. Clin. Oncol., 2016, 13, 242-254.

103 L. Simon, A. Imane, K. K. Srinivasan, L. Pathak and I. Daoud, In Silico Drug-Designing Studies on Flavanoids as Anticolon Cancer Agents: Pharmacophore Mapping, Molecular Docking, and Monte Carlo Method-Based QSAR Modeling, Interdiscip. Sci.: Comput. Life Sci., 2017, 9(3), 445-458.

104 T. Atul, T. Abhishek and A. Priya, CancerPPD: a database of anticancer peptides and proteins, Nucleic Acids Res., 2015, 43, D837-D843.

105 R. Kumar, et al. CancerDR: Cancer Drug Resistance Database, Sci. Rep., 2013, 1445.

106 T. Weiyang, L. Bohui, G. Shuo, et al. CancerHSP: anticancer herbs database of systems pharmacology, Sci. Rep., 2015, 5, 11481.

107 V. Umashankar, S. Nandhitha and P. Kalabharath, InPACdb - Indian plant anticancer compounds database, Bioinformation, 2009, 4(2), 71-74.

108 J Gollub, C. A. Ball, G. Binkley, J. Demeter, D. B. Finkelstein, J. M. Hebert, T. Hernandez-Boussard, H. Jin, M. Kaloper, J. C. Matese, M. Schroeder, P. O. Brown, D. Botstein and G. Sherlock, The Stanford Microarray Database: data access and quality assessment toolsOxford Journals Life Sciences, Nucleic Acids Res., 2003, 31(1), 94-96.

109 Y. Bernard, C. Konstantina and R. Sophie, Statistical Analysis of GEO Datasets, Gene Technol., 2014, 4, 1.

110 F. J. Romero-Duran, N. Alonso, M. Yanez, O. Caamano, X. Garcia-Mera and H. Gonzalez-Diaz, Brain-inspired cheminformatics of drug-target brain interactome, synthesis, and assay of TVP1022 derivatives, Neuropharmacology, 2016, 103, 270-278.

111 Forum on Neuroscience and Nervous System Disorders, Board on Health Sciences Policy; Institute of Medicine, Improving and Accelerating Therapeutic Development for Nervous System Disorders: Workshop Summary, National 
Academies Press (US), Washington (DC), 2014, Drug Development Challenges.

112 Personalized Medicine 101: The Challenges, Personalized Medicine Coalition, Retrieved April 26, 2014.

113 H. E. K. Stefan, M. E. MJuliana and J. M. L. David, Challenges and responses in human vaccine development, Curr. Opin. Immunol., 2014, 28, 18-26.

114 A. Speck-Planche and M. N. D. S. Cordeiro, De novo computational design of compounds virtually displaying potent antibacterial activity and desirable in vitro ADMET profiles, Med. Chem. Res., 2017, 26(10), 2345-2356.

115 V. V. Kleandrova, J. M. Ruso, A. Speck-Planche and M. N. Dias Soeiro Cordeiro, Enabling the Discovery and Virtual Screening of Potent and Safe Antimicrobial Peptides. Simultaneous Prediction of Antibacterial
Activity and Cytotoxicity, ACS Comb. Sci., 2016, 18(8), 490498.

116 A. Speck-Planche and M. N. D. S. Cordeiro, Simultaneous virtual prediction of anti-Escherichia coli activities and ADMET profiles: A chemoinformatic complementary approach for high-throughput screening, ACS Comb. Sci., 2014, 16(2), 78-84.

117 S. T. Park and J. Kim, Trends in Next-Generation Sequencing and a New Era for Whole Genome Sequencing, Int. Neurourol. J., 2016, 20, 76-83.

118 L. Barzon, E. Lavezzo, V. Militello, S. Toppo and G. Palù, Applications of next-generation sequencing technologies to diagnostic virology, Int. J. Mol. Sci., 2011, 12(11), 78617884.

119 S. Tony, Immunology of Bats and Their Viruses: Challenges and Opportunities, Viruses, 2014, 6, 4880-4901. 